Background Sport specialization can impact physical and mental aspects of the individual athlete.

Objective To evaluate the relationship between sports participation history, success, health status, and injury.

Design Recall Survey.

Setting Major League Baseball Team.

Patients (or Participants) 107 Major League Baseball Players.

Interventions (or Assessment of Risk Factors) A posterori assessment of years of single sport participation, overall participation in sport, current age.

Main Outcome Measurements Determination of the impact of duration of sports participation and specialization on injury, adjusting for age.

Results $75 \%$ of the athletes reported playing at least one other sport competitively; primarily basketball. The average years playing baseball was (19) and the average years specializaing in baseball was (9). 80 (75\%) reported being born and raised in the U.S. $12 \%$ reported that their mother played high-level sports while $37 \%$ reported that their father played high-level sports with $63 \%$ of those playing baseball. 7 injuries that prohibited participation for at least 12 weeks were reported. The greatest proportion of active athletes who were injured occured at $16 \%$ (age 24). A Cox model with a time varying covariate representing specialization, adjusted for the age athletes started baseball, showed no significant relationship between specialization and injury. The time scale used in the model was years since an athlete started baseball. On average specialization had a substantial impact on their elite success was 7 on a scale of 0 (No impact) to 10 (Extreme Impact). Compared to non-elite athletes respondents indicated that on average their physical, emotional health, and general well being was better.

Conclusions Elite athletes perceive that specialization is important to playing at a high level. They are physically, emotionally healthier and have a greater well being that non-athletes their age. A time to event modeling to determine the impact of duration of sports participation and specialization on injury, adjusting for the age players started baseball did not demonstrate a significant relationship between specialization and injury.

\section{US YOUTH SOCCER COACHES DO NOT POSSESS ADEQUATE KNOWLEDGE OF NON-CONTACT ACL INJURIES AND INJURY PREVENTION PROGRAMS}

Shelly Fetchen DiCesaro. California University of Pennsylvania, California, USA

\subsection{6/bjsports-2021-IOC.329}

Background Non-contact anterior cruciate ligament (NC-ACL) injuries have been shown to occur more often for female soccer players as opposed to their male counterparts; however, it has been demonstrated that prevention programs may significantly decrease these injuries. US Youth Soccer players are predominately coached by volunteers who may not have an understanding of preventative strategies.

Objective To assess understanding of NC-ACL injuries and the implementation of prevention programs in US Youth Soccer coaches.

Design Cross-sectional descriptive survey.

Setting Blind online survey administered to volunteer US Youth Soccer coaches.
Patients (or Participants) Individuals over 18 years of age who were registered with US Youth Soccer as a volunteer coach received the survey. The exact number of email invitations to participate opened was unattainable.

Interventions (or Assessment of Risk Factors) A 51 question survey was used to determine knowledge regarding NCACL injuries. Survey questions were constructed from a literature review and expert experience.

Main Outcome Measurements The main outcome measure is the percentage of correct answers on the survey with a score of $75 \%$ deemed knowledgeable.

Results Three hundred and fifty-six (356) surveys were accessed from the email invitation with 330 suitable for data analysis. Participants scored an average of 18 questions incorrect for a total knowledge score of $63.98 \%$, below the required $75 \%$. Additionally, scores for males $(n=277$, score $=$ $63.83 \%)$ and females $(n=53$, score $=64.70)$ did not differ significantly as analyzed by t-test with a $\mathrm{p}=0.47$.

Conclusions US Youth Soccer coaches do not possess adequate knowledge of NC-ACL injuries and implementation of prevention strategies. Further analysis may elicit significance between scores specific to experience, length of time coaching and previous injury prevention program education. Coaches and their players would benefit from mandatory standardized education and strategies for implementation into lesson plans and practice.

\section{COACH EDUCATION AS A STRATEGY TO IMPROVE ADHERENCE TO ACL INJURY PREVENTION PROGRAMS: A CLUSTER-RANDOMIZED CONTROLLED TRIAL}

Daphne Ling, Caroline Boyle, Brandon Schneider, Joseph Janosky, James Kinderknecht, Robert Marx. Hospital for Special Surgery, New York, USA

\subsection{6/bjsports-2021-IOC.330}

Background Despite evidence of its efficacy, ACL injury prevention programs have had limited success in convincing coaches to perform the recommended exercises.

Objective We evaluate a coach education workshop that trains coaches on how to implement an injury prevention program and measure the impact of this strategy on adherence rates.

Design Cluster-randomized controlled trial.

Setting High school sports teams.

Participants Eight high schools were recruited and randomized 1:1 to the intervention or control arm. The intervention schools contained 12 teams (5 girls), and the control schools contained 10 teams (4 girls). The sports included basketball, volleyball, track and field, baseball/softball, and lacrosse.

Interventions Our institution's Sports Safety Program has developed a 60-minute education workshop aimed at coaches that teaches them how to provide exercise instruction and to correct movement deficiencies using feedback cues. The schools in the control arm received print materials on recommended exercises.

Main Outcome Measurements Coach adherence was the main outcome. Eight data collectors, who were blinded to the team's assignment, were trained to observe a team's practice or game 3 times a week. At each session, they completed a survey to identify the exercise and then answered whether the coach 1) provided exercise instructions and 2) provided feedback cues. 
Results A total of 399 practice or game sessions were observed over 2 sport seasons (62\% in intervention schools).A greater proportion of coaches in the intervention group provided alignment cues to correct improper technique compared to coaches in the control group [difference $=0.04$ (95\% CI: $0.01,0.07, \mathrm{p}=0.006]$. There was a similar proportion of coaches in the intervention and control groups who provided exercise instructions [difference $=0.01$ (95\% CI: $-0.02,0.04$ ), $\mathrm{p}=0.44]$.

Conclusions One barrier to adoption of injury prevention programs may be the coach's lack of knowledge on how to implement a warm-up routine that is effective at reducing ACL injuries. A coach education workshop may lead to actual behavior change in practice.

\section{THE RELATIONSHIP BETWEEN THE INJURIES IN ELEMENTARY PE AND THE STAGES OF TEACHER PROFESSIONAL DEVELOPMENT}

Yuki Nakamura. Hokuriku university, Kanazawa, Japan

\subsection{6/bjsports-2021-IOC.331}

Background In Japan, the ministry has emphasized that safety management is important for PE instructions.

Objective The purpose of this study is to clarify the relationship between the injuries in elementary PE and the stages of teacher professional development.

Design The method of this study is to collect the number of injuries in elementary $\mathrm{PE}$ in each professional development stage by the record of Japanese school health room. These collected data are classified into three types of injuries: Open wound, Closed wound, Other wound. The survey was conducted in 2014.

Setting Elementary school of physical education.

Patients (or Participants) The subjects were nine Japanese elementary schools that received research cooperation. The number of participants was 54 teachers (20 novice teachers, 15 experienced teachers, 19 expert teachers).

Interventions (or Assessment of Risk Factors) The independent variables were the three stages of teacher professional development.

Results 1) When focusing on the total number of injuries, there were no significant differences among three professional development stages $(p<.05)$. 2) When focusing on the total number of injuries, there were no significant differences between male novice teachers, female novice teachers, male experienced teachers, female experienced teachers, male expert teachers and female expert teachers $(\mathrm{p}<.05)$. When focusing on the proportion of three types of injuries, there were significant differences among three professional development stages $(\mathrm{p}<.05)$. Closed wound were more common in the experienced teacher stage and less common in the expert teacher stage. On the other hand, Open wound was more common in the Expert teacher stage and less common with Novice teachers and Experienced teachers.

Conclusions In particular, it is suggested that the quality of injury changes from serious injuries to minor injuries between Experienced teachers and Expert teachers. That is, it implicates that professional development of safety management in $\mathrm{PE}$ is different between Experienced teacher and Expert teacher.

\section{5 MOVE HEALTHY-THE IDENTIFICATION OF CURRENT NATIONAL INJURY PREVENTION PROGRAMS AND BELIEFS OF COACHES AND YOUTH REGARDING INJURY PREVENTION IN 6 EUROPEAN COUNTRIES}

${ }^{1}$ Joske Nauta, ${ }^{2}$ Johan de Jong, ${ }^{3}$ Kristine De Martelaer, ${ }^{4}$ Paul Dragos, ${ }^{2}$ Remo Mombarg, ${ }^{5}$ Danielle Nørager Johansen, ${ }^{5}$ Thomas Skovgaard, ${ }^{4}$ Paul Szabo-Alexi, ${ }^{1}$ Evert Verhagen,

${ }^{2,6}$ Anne Benjaminse. ${ }^{1}$ Amsterdam UMC, Amsterdam, Netherlands; ${ }^{2}$ Hanze University of Applied Sciences, Groningen, Netherlands; ${ }^{3}$ Vrije Universiteit Brussel, Brussel, Belgium; ${ }^{4}$ University of Oradea, Oradea, Romania; ${ }^{5}$ University of Southern Denmark, Odense, Denmark; ${ }^{6}$ University Medical Center Groningen, Groningen, Netherlands

\subsection{6/bjsports-2021-IOC.332}

Background Despite the importance of sports injury prevention in youth, no broad scale approaches that work in real-life situations with significant positive effects exist. Main reasons for this are poor uptake and maintenance of current sports injury prevention exercises.

Objective In order to improve uptake of sports injury prevention routines, this project set out to: 1 . identify the specifics of current injury prevention programs within 6 European countries, and 2: to establish wishes and needs regarding injury prevention of the end-users (sport coaches, physical educators and youth) within 6 European countries.

Design Semi-structured interviews and focus group sessions. Setting Youth sports teams and physical education (PE) classes. Patients (or Participants) Interviews and focus group sessions were performed within 6 participating countries (Belgium, Denmark, Lithuania, Romania, The Netherlands, United Kingdom). National stakeholders were interviewed to identify injury prevention programs. The program owners of the identified programs were then individually interviewed. The focus group sessions were organized with youth basketball and soccer players and PE pupils. Separate focus group sessions were organized for basketball/soccer coaches and physical educators.

Interventions (or Assessment of Risk Factors) This qualitative study provided input for the development of a freely available ICT based platform with video material of routines designed to prevent sports injuries in youth.

Main Outcome Measurements Overview of country specific results of interviews and focus groups.

Results This study will describe the current availability of national injury prevention programs within 6 European countries. The results of the focus group sessions will establish the differences in beliefs regarding injury prevention in 6 European countries regarding injury prevention.

Conclusions In the coming year, the Move Healthy project will use the qualitative results of this study to develop a freely available ICT based platform with video material of routines developed to prevent sports injuries in youth.

\section{SHINING A LIGHT ON INJURY STATUS: DEVELOPING AN EFFECTIVE METHOD TO MONITOR PLAYER AVAILABILITY AND IMPROVE COMMUNICATION BETWEEN COACHES AND MEDICAL STAFF}

\footnotetext{
${ }^{1,2}$ Ciarán Purcell, ${ }^{1,2}$ Catherine Blake, ${ }^{1,2}$ Ulrik McCarthy Persson, ${ }^{1,2}$ Sinead McMahon,

${ }^{1,2}$ Caitriona Cunningham, ${ }^{1,2}$ Frank Kenny. ' UCD Physiotherapy Hub, Dublin, Ireland; ${ }^{2}$ UCD School of Public Health, Physiotherapy and Sport Science, Dublin, Ireland
}

10.1136/bjsports-2021-IOC.333 